\title{
lontophoresis-assisted pediatric transdermal delivery of ropivacaine to enhance anesthetic effect
}

\author{
Liqun Yu, Yanna Liu, Miao Sang* \\ Department of Pediatrics, The Second People's Hospital of Dong ying, Shandong Province 257300, China
}

*For correspondence: Email: sangmiao666@sina.com

Sent for review: 16 June 2020

Revised accepted: 15 March 2021

\begin{abstract}
Purpose: To formulate novel transdermal patches of ropivacaine (RPV) and enhance its anesthetic activity using iontophoresis technique.

Methods: Solvent casting method was used for the development of the transdermal patches with the aid of hydroxypropyl methylcellulose (HPMC) and polyvinyl alcohol (PVA) polymers. The patches were characterized for appearance, clarity, smoothness, thickness, average weight of patches, folding endurance, RPV uniformity, moisture absorption and tensile strength. The anesthetic effect of ropivacaine patches were evaluated in a healthy pediatric population divided into two groups. One group was merely applied with the patch while the other group received the patch using LidoSite applicator. The anesthetic effect was assessed based on hot pain, cool sensation and warm sensation. Results: The patches were smooth and transparent, about $0.29 \mathrm{~mm}$ in thickness, having a tensile strength of $0.51 \mathrm{~kg} / \mathrm{cm}^{2}$ and showed in-vitro drug release of 68.94 to $96.83 \%$. The formulation did not exert any undesirable local skin effects, and produced minimum pain intensity. The depth of anesthesia was higher for the patch formulation with iontophoresis than for the conventional system.

Conclusion: The findings point to the superiority of RPV transdermal patches when applied with iontophoretic device as against the conventional application.
\end{abstract}

Keywords: Iontophoresis, Transdermal drug delivery, Ropivacaine, Local anesthesia, hydroxypropyl methylcellulose (HPMC), polyvinyl alchohol (PVA), LidoSite

\begin{abstract}
This is an Open Access article that uses a fund-ing model which does not charge readers or their institutions for access and distributed under the terms of the Creative Commons Attribution License (http://creativecommons.org/licenses/by/4.0) and the Budapest Open Access Initiative (http://www.budapestopenaccessinitiative.org/read), which permit unrestricted use, distribution, and reproduction in any medium, provided the original work is properly credited.

Tropical Journal of Pharmaceutical Research is indexed by Science Citation Index (SciSearch), Scopus, International Pharmaceutical Abstract, Chemical Abstracts, Embase, Index Copernicus, EBSCO, African Index Medicus, JournalSeek, Journal Citation Reports/Science Edition, Directory of Open Access Journals (DOAJ), African Journal Online, Bioline International, Open-J-Gate and Pharmacy Abstracts
\end{abstract}

\section{INTRODUCTION}

Amongst various drug delivery systems available today, transdermal drug delivery system (TDDS) has proved its patient compliance with remarkable improvement in patients' quality of life (QOL) because of its prime advantages for elderly and bedridden patients so as to avoid first pass metabolism [1]. The other clinical benefits include controlled drug delivery with maintenance of steady state plasma concentrations without pain. Stratum corneum which is the uppermost lipidic layer of skin acts as the barrier for the absorption of highly polar and hydrophilic drugs. The penetration or absorption of such drugs can be improved by using various penetration enhancers and physico-mechanical techniques [2]. Nowadays, iontophoresis (IP) has been emerged as very useful technique which involves transfer of charged molecules via biological 
membranes and tissues using a mild electrical current applied externally [3]. The voltage applied in IP is low $\left(0.5 \mathrm{~mA} / \mathrm{cm}^{2}\right)$ as compared to electrophoresis. IP utilizes application of two oppositely charged electrodes across skin so as to drive drug molecule having similar charges. Therefore, the effects obtained in this type of system are mostly concentration dependent [4].

The method showed various advantages in transdermal delivery system including patient compliance in patch application, minimum adverse effects with enhanced drug permeation and bioavailability [5]. This technique has also been widely utilized to transport the drugs to enhance anesthetic effect of lignocaine, to treat onychomycosis, bursitis and tendonitis etc [6]. Ropivacaine (RPV) is potential amide type local anesthetic agent having similar therapeutic as well as chemical properties to that of Bupivacaine [7]. RPV showed very lower cardiovascular and central nervous system related toxicities as compared to the Bupivacaine after intravenous infusion. In healthy human volunteers RPV was found to be at least $25 \%$ less toxic when compared to Bupivacaine [8]. RPV showed distinct separation of motor and sensory block due to the slight lower lipid solubility in clinical trials and also allowed an earlier motor blockade recovery in relation to Bupivacaine. Due to the aforementioned advantages, RPV is well suited for the postoperative analgesia [9]. The objective of current research work was to formulate iontophoresisassisted pediatric RPV embedded transdermal patches to enhance anesthetic effect.

\section{EXPERIMENTAL}

\section{Materials}

Ropivacaine was obtained from Shouguang Fukang Pharmacy Factory (Shandong, China), hydroxy propyl methyl cellulose (HPMC), polyvinyl alcohol (PVA) were purchased from Sigma Aldrich, USA, dibutyl pthalate, acetic acid, and, methanol were purchased from Shanghai Chemical Co. (Shanghai, China). LidoSite ${ }^{\mathrm{TM}}$ Controller system purchased from Amsterdam pharmacy (USA).

\section{Formulation of ropivacaine patches (RPV patch)}

Solvent casting method was used to prepare transdermal patch of RPV. The polymeric solution was prepared by dissolving $5 \%$ PVA and $0.5 \%$ HPMC in distilled water (Solution A) under continuous stirring at $40-45^{\circ} \mathrm{C}$. The drug solution was prepared by dissolving RPV in purified water under continuous magnetic stirring (solution B). The solution A and solution B were mixed with continuous gentle shaking with addition of $0.3 \%$ dibutyl phthalate as plasticizer. This dispersion was poured on glass Petri dish with known diameter. The Petri dish was kept to dry in an oven at $50{ }^{\circ} \mathrm{C}$ for $5-6 \mathrm{~h}$. The underside of the Petri dish was previously partitioned into $2.5 \mathrm{~cm} \times 2.5 \mathrm{~cm}$ sized squares. The dried patches were peeled off slowly, cut in to uniform pieces and stored in a desiccator for pending analysis [10]. Similarly, electrolyte reservoir containing sodium chloride, glycerin, preservative (isobutyl-p-hydroxybenzoate) was prepared [11].

\section{Characterization of RPV patch}

All patches were observed visually for their appearance, clarity, smooth nature and colour.

\section{Thickness}

The average thickness of patch was calculated by measuring the thickness at 4 various sites using micrometer screw gauze [12].

\section{Weight}

The average weight of patch was determined by weighing 10 patches on calibrated weighing balance [12].

\section{Folding endurance}

The physical strength of the patch was determined by using folding endurance test. The patch was folded repeatedly at same point till it broken. The numbers of folds were counted at specific point without break up of patch was considered as folding endurance strength [12].

\section{Content uniformity}

Uniform distribution of RPV was determined by using this test. Ten uniform patches were dissolved in methanolic solution under stirring and resulting solution was filtered through 0.3 micron filter to get clear solution. These solutions were analyzed at $320 \mathrm{~nm}$ by ultra violet spectrophotometer [12].

\section{Assessment of moisture absorption}

The initial weight (W1) of randomly selected 5 patches were recorded and kept in desiccator. The final weight (W2) was determined after period of 5 days. The percentage of moisture absorbed was calculated using Eq 1 [12]. 
Moisture uptake $(\%)=\{(\mathrm{W} 1-\mathrm{W} 2) / \mathrm{W} 1\} 100 \ldots$ (1)

\section{Evaluation of tensile strength}

The mechanical property of the patch was determined based on its tensile strength using universal tensile strength testing apparatus (Hounsfield, Slinfold, Horsham, U.K) [12].

\section{Skin permeability study}

In-vitro skin permeability was conducted on human cadaver skin using modified glass cell to which skin portion was connected. A glass tube (as donor compartment) was dipped into glass beaker containing $\mathrm{pH} 7.4$ phosphate buffer as receptor medium. Patch measuring $1 \mathrm{~cm}^{2}$ was mounted on membrane. The steady temperature of $37 \pm 0.5^{\circ} \mathrm{C}$ was maintained on the magnetic at $600 \mathrm{rpm}$. The $1 \mathrm{~mL}$ aliquots were removed at interval of 1 hour for the period of 9 hour and replenished equal amount of fresh buffer to maintain the sink condition. Using spectrometric analysis ant $320 \mathrm{~nm}$ samples were analyzed to determine the drug released [12].

\section{Selection of population for trial}

The study was conducted as per approval of human ethical committee of Department of Pediatrics, The Second People's Hospital of Dong ying, Shandong Province, 257300, China. (Approval no: KYLL-202012CR). The study was conducted as per guidelines of The Council for International Organizations of Medical Sciences (CIOMS) [fourth version of the CIOMS Guidelines (2016), Guideline 17 [13]]. The protocol of the trial to be conducted was made clear to parent/guardian (as trials were conducted on children). The randomized, controlled trial was conducted with 20 subjects. None of the subject was excluded from study due to reason of allergy or non-compliance of patient to trial.

\section{Application of LidoSite system}

The subjects were selected randomly and divided in to two groups. Group I subjects were applied with Lidosite TM system while group II were applied without any system. A patch containing ropivacaine $30 \mathrm{mg}$ was applied directly to the skin of group II subjects while patch with LidoSite system was applied to group I subjects. The patch samples used were similar in physical and chemical properties like drug content, weight uniformity, thickness etc (Table 1).
Table 1: Distribution of the sample population based on age, sex, body weight and pre-medication

\begin{tabular}{lcc}
\hline Variable & Group I & Group II \\
\hline Boys/girls (mean \pm SD) & $6 / 4$ & $7 / 3$ \\
Weight $(\mathrm{kg}$, mean & $28.2 \pm 1.02$ & $26.09 \pm 0.89$ \\
$\pm S D)$ & & \\
Age $(\mathrm{yr}$, mean $\pm S D)$ & $6.1 \pm 1.02$ & $7.21 \pm 1.25$ \\
Pre-medication & None & None \\
Application & Lidosite $^{\mathrm{TM}}$ & $\mathrm{NA}$ \\
\hline
\end{tabular}

\section{Determination of end-point of experiment- tation}

Various end point parameters like hot pain sensation, cool sensation and warm sensation determined for duration of anesthesia under supervision of skilled medical professional. The end points were observed at $0,15,30,45,60$ and $120 \mathrm{~min}$. The thermal sensation was checked at central part of treated area. Pain sensation was measured using depth of anesthesia. The insulating material was filled in the gap of electrode which was having alternating polarity, was used to check stimuli from lower to higher order. The temperature was kept on changing as $1{ }^{\circ} \mathrm{C} / \mathrm{s}$ as per direction of device flow. A 27-gauge short needle was inserted perpendicular to skin in one direction slowly and smoothly in order to measure anesthesia. The first sensation of pain was marked on visual scale in Figure 1. The needle was removed and the depth of needle insertion was marked. Any visible signs like redness, swelling etc were observed on treated area.

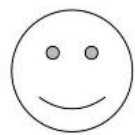

A

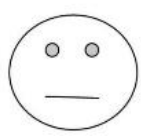

B

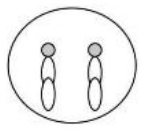

C
Figure 1: Visual scale showing intensity of pain from "no pain" for face A, "medium pain" for face B and "unbearable pain" for face $C$

\section{Measurement of drug absorption}

The blood samples were collected from both groups (group I and II) at predetermined time interval of $0,10,20,30,45$ and $60 \mathrm{~min}$. The amount of RPV in blood samples were determined using a validated bioanalytical method based in liquid chromatography-mass spectrometry (LC-MS). The differences in concentration of RPV in both groups showed the effect of iontophoresis on TDDS. 


\section{Statistical analysis}

Statistical analysis of the experimental work was carried out using Design Expert 6.0.8 portable Stat-Ease, Inc software and compared using Student $\mathrm{t}$-test. Data are presented as mean \pm $\mathrm{SD}$. The results were considered statistically significant at $p<0.05$.

\section{RESULTS}

RPV transdermal patches were successfully formulated by solvent casting technique using HPMC, PVA and dibutyl phthalate as polymers and plasticizers respectively. The developed patches showed pharmaceutical compliance with respect to different physicochemical parameters. The observations for evaluation tests were shown in Table 2.

Table 2: Evaluation parameters of patches

\begin{tabular}{ll}
\hline Parameter & Observation \\
\hline Appearance & Smooth, transparent \\
Thickness & $0.29-0.48 \mathrm{~mm}$ \\
Moisture content & $1.56-2.34 \%$ \\
Folding endurance & $145-175$ times \\
Mean weight & $0.20-0.24 \mathrm{~g}$ \\
In-vitro release & $68.94-96.83 \%$ \\
\hline
\end{tabular}

The surface of patches were found to be smooth and transparent in appearance with no haziness with excellent surface characteristics. The patches were found to be $0.29 \pm 0.005 \mathrm{~mm}$ to $0.48 \pm 0.003 \mathrm{~mm}$ in thickness with excellent tensile strength of $0.51 \pm 0.006$ to $0.64 \pm 0.002$ $\mathrm{kg} / \mathrm{cm}^{2}$. The in-vitro RPV release from patch was found to be $68.94 \%$ to $96.83 \%$ in release media of $\mathrm{pH} 7.4$ buffer up to $9 \mathrm{hrs}$. (Figure 2) The results were found statistically significant with $P$ $<0.05$.

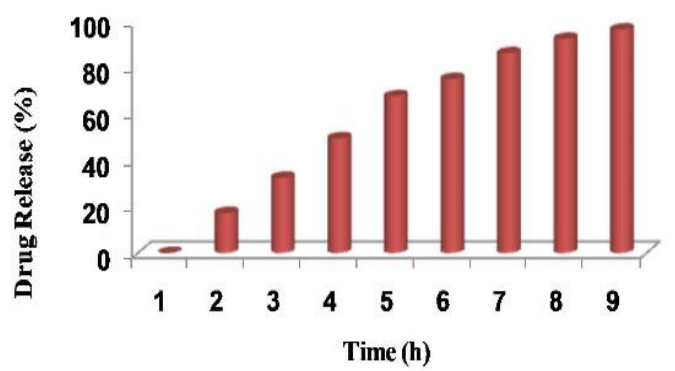

Figure 2: In vitro permeation of RPV from transdermal patch at $9 \mathrm{~h}$

The moisture content of the patches was 1.56 to $2.34 \pm 0.24 \%$ while folding endurance was $145.21 \pm 0.2$ to $175 \pm 0.29$. The mean weight of the patches was $0.20 \pm 0.2$ to $0.24 \pm 0.18 \mathrm{~g}$.
The subjects undergoing treatment (in both group) were reluctant to show any kind of signs of swelling, itching or edema while slight amount of tingling sensation was observed which was considered as normal response as shown in Table 3.

Table 3: Local effect on subjects' skin

\begin{tabular}{lccc}
\hline Group & Swelling & Redness & Itching \\
\hline I & 0 & 0 & $0-1$ \\
II & 0 & 0 & 0 \\
\hline 0 = Negative; & $0-1$ = Slight skin irritation; & 1-2 = Severe \\
skin effects (all signs visible) & &
\end{tabular}
skin effects (all signs visible)

The time required for subjects in each group to feel pain is shown in Table 4. The pain intensity for group without LidoSite system (Group II) was found to be higher than those in group with Lidosite TM system (Group I) $(p<0.05)$. The intensity of pain vanishes faster in Group I than in Group II. These observations clearly indicated the enhanced anesthetic effect of RPV patches due to Lidosite TM system incorporating iontophoresis in Group I.

Table 4: Comparative pain intensity scores among two groups using VAS

\begin{tabular}{llcccccc}
\hline \multicolumn{2}{l}{ Time (min) } & $\mathbf{0}$ & $\mathbf{1 0}$ & $\mathbf{2 0}$ & $\mathbf{3 0}$ & $\mathbf{4 5}$ & $\mathbf{6 0}$ \\
\hline $\begin{array}{l}\text { Pain } \\
\text { intensity }\end{array}$ & Group & 8.1 & 7.8 & 5.7 & 3.7 & 00 & 00 \\
score & $\begin{array}{l}\text { Group } \\
\text { II }\end{array}$ & 8.3 & 8.0 & 7.6 & 6.1 & 4.9 & 2.9 \\
\hline
\end{tabular}

The initial score for pain intensity at 0 was numerically same at least comparable but as time passes the pain in subjects with Group I go on decreasing than Group II. After $45 \mathrm{~min}$, subjects in Group I reported "no pain" while Group II were still complaining about severe to moderate pain $(p<0.05)$. So, this numerical difference in intensity score shows the superiority of transdermal patch when administered with aid of iontophoresis (Figure 2).

The depth of anesthesia is unit to measure distance in $\mathrm{mm}$ up to which needle can inserted. So, the distance of needle prick is seen higher in Group I than in Group II $(p<0.05)$. This observation also proved the superiority of iontophoresis assisted TDDS of RPV (Figure 3).

\section{DISCUSSION}

Our research group has attempted to develop iontophoresis assisted TDDS of RPV to enhance the anesthetic effect of active molecule. The major barrier for the transdermal delivery is the stratum corneum which reduces absorption of active metabolite through transdermal route [14]. 


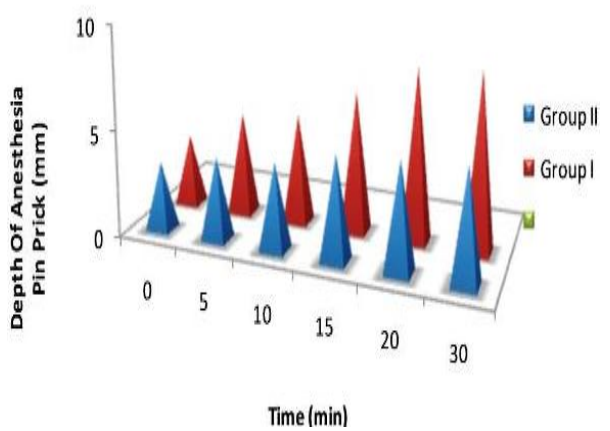

Figure 3: Comparative depth of anesthesia, with Group I showing greater efficiency

The use of chemicals as permeation enhancers might be harmful for the skin so the iontophoresis is ideal in such scenario [15]. The enhancement in the anesthetic activity through transdermal patches was due to the increased permeation of RPV using electro potential energy generated during iontophoresis. The transport of drugs via skin was thought to be improved with help of iontophoresis as after ionization drugs acquire negative charges. The externally applied electrical potential causes an increase in ionic movement across biological membranes. The concentration of active molecule permeated through skin barrier has direct relation with respect to application of electrical charge and time span of application $[16,17]$.

Various researchers have used iontophoresis to maximize the drug permeation through skin and enhanced the therapeutic effect of active molecule in various diseased conditions. Some study has shown that when external current is applied for prolong period of time generate heat energy which will fluidized skin lipids in order to change the natural integrity of skin and therefore increasing permeability of skin [18]. This phenomenon explains sufficiently the reason for increased RPV action by applying lontophoretic drug delivery system for prolonging anesthetic effect. Kimiko et al developed iontophoresis coupled transdermal estradiol nanoparticles for efficient treatment and management of osteoporosis, These authors found that nanoparticulate system coupled with iontophoresis was useful for osteoporosis treatment [19]. Similarly, Szekanecz et al developed iontophoresis assisted topical delivery of calcium and phosphates microparticles for the osteoporosis condition which was found comparable with estrogen therapy [20]. Likewise, Makino et al enhanced the permeation of estradiol nanoparticles utilizing iontophoresis and concluded that permeation of estradiol could be enhanced by employing nanoparticles and iontophoresis [21]. The foregoing studies indicate the importance and efficiency of iontophoresis in permeation enhancement of active molecules in various diseased conditions. In the present work, the therapeutic efficacy of RPV has been successfully enhanced using iontophoresis; this is an advantage in prolonging anesthesia.

\section{CONCLUSION}

The findings of this study show that iontophoresis-mediated RPV TDDS is a potential alternative to the conventional RPV delivery system for the enhancement of anesthetic activity in pediatrics.

\section{DECLARATIONS}

\section{Acknowledgement}

Authors are grateful to Department of Pediatrics, The Second People's Hospital of Dong ying, Shandong Province, China for providing the facilities to conduct this research.

\section{Conflict of interest}

No conflict of interest is associated with this work.

\section{Contribution of authors}

We declare that this work was done by the authors named in this article and all liabilities pertaining to claims relating to the content of this article will be borne by the authors.

\section{Open Access}

This is an Open Access article that uses a funding model which does not charge readers or their institutions for access and distributed under the terms of the Creative Commons Attribution License (http://creativecommons.org/licenses/by/ 4.0) and the Budapest Open Access Initiative (http://www.budapestopenaccessinitiative.org/rea d), which permit unrestricted use, distribution, and reproduction in any medium, provided the original work is properly credited.

\section{REFERENCES}

1. Al-Kassas R, Wen J, Cheng AE, Kim AM, Liu SS, Yu J. Transdermal delivery of propranolol hydrochloride through chitosan nanoparticles dispersed in mucoadhesive gel. Carbohydr. Polym 2016; 153: 176186. 
2. Liu X, Testa B, Fahr A. Lipophilicity and its relationship with passive drug permeation. Pharm. Res 2011; 28(5): 962-977.

3. Schuetz YB, Naik A, Guy RH, Kalia YN. Emerging strategies for the transdermal delivery of peptide and protein drugs. Expert Opin Drug Deliv 2005; 2(3): 533548.

4. Calatayud-Pascual MA, Balaguer-Fernández C, SernaJiménez CE, Del Rio-Sancho S, Femenía-Font $A$, Merino V, López-Castellano A. Effect of iontophoresis on in vitro transdermal absorption of almotriptan. Int. J. Pharm 2010; 416(1): 189-194.

5. Chang SL, Hofmann GA, Zhang L, Deftos LJ, Banga AK. Transdermal iontophoretic delivery of salmon calcitonin. Int. J. Pharm 2000; 200(1): 107-113.

6. Kalia YN, Naik A, Garrison J, Guy RH. Iontophoretic drug delivery. Adv Drug Deliv Rev 2004; 56(5): 619-658.

7. Ernberg M, Kopp S. Ropivacaine for dental anesthesia: a dose-finding study. J. Oral Maxillofac. Surg 2002; 60(9): 1004-1010.

8. Martini E, Cavallini GM, Campi L, Lugli N, Neri G, Molinari P. Lidocaine versus ropivacaine for topical anesthesia in cataract surgery. J Cataract Refract Surg 2002; 28(6): 1018-1022.

9. Kawata T, Homma M, Kakiuchi $Y$, Inomata S, Miyabe $M$, Kobayashi D, Morimoto $Y$, Kohda Y. Liquid chromatographic determination of plasma ropivacaine for assessing pharmacokinetics of the viscous preparation. Biol. Pharm. Bull 2005; 28(12): 2271-2273.

10. Pierce MW. Transdermal delivery of sumatriptan for the treatment of acute migraine. Neurotherapeutics 2010; 7(2): 159-163.

11. XiaoLi, TianzuoLi. Iontophoretic delivery of transdermal patches containing lignocaine with clonidine and epinephrine in healthy volunteers: Effect of concentration of epinephrine and clonidine on local anesthesia. Biomedical Research 2017; 28(15): 66406645.

12. Dey $S K$, De $P K$, Sen $T$, Shankar $V$, Banerjee $U$. Formulation and in vitro evaluation of transdermal matrix patches of Diclofenac sodium. J. Pharm. Res 2011; 4(10): 3593-3596.
13. International Ethical Guidelines for Health-related Research Involving Humans. The Council for International Organizations of Medical Sciences (CIOMS): The fourth version of the CIOMS Guidelines (2016); Guideline 17. Available from: https://cioms.ch/wp-content/uploads/2017/01/WEBCIOMS-EthicalGuidelines.pdf

14. Sugawara $T$, Iwamoto $N$, Akashi M, Kojima T, Hisatsune $J$, Sugai $M$, Furuse M. Tight junction dysfunction in the stratum granulosum leads to aberrant stratum corneum barrier function in claudin-1-deficient mice. J. Dermatol. Sci 2013; 70(1): 12-18.

15. Dhote V, Bhatnagar $P$, Mishra PK, Mahajan SC, Mishra $D K$. Iontophoresis: a potential emergence of a transdermal drug delivery system. Sci. Pharm 2012; 80(1): 1-28.

16. Cheng C, Jiang G, Simon GP, Liu JZ, Li D. Low-voltage electrostatic modulation of ion diffusion through layered graphene-based nanoporous membranes. Nat. Nanotechnol 2018; 13(8): 685-690.

17. Banga AK, Bose S, Ghosh TK. Iontophoresis and electroporation: comparisons and contrasts. Int. J. Pharm 1999; 179(1): 1-9.

18. Sala M, Diab R, Elaissari A, Fessi H. Lipid nanocarriers as skin drug delivery systems: Properties, mechanisms of skin interactions and medical applications. Int. J. Pharm 2018; 535(1-2): 1-7.

19. Takeuchi I, Fukuda K, Kobayashi S, Makino K. Transdermal delivery of estradiol-loaded PLGA nanoparticles using iontophoresis for treatment of osteoporosis. Biomed. Mater 2016; 27(5): 475-483

20. Gomez I, Szabó A, Pap Jr L, Pap L, Boda K, Szekanecz $Z$. In vivo calcium and phosphate iontophoresis for the topical treatment of osteoporosis. Phys. Ther. 2012; 92(2): 289-297.

21. Tomoda $K$, Watanabe A, Suzuki K, Inagi $T$, Terada $H$, Makino K. Enhanced transdermal permeability of estradiol using combination of PLGA nanoparticles system and iontophoresis. Colloids Surf B Biointerfaces 2012; 1(97): 84-89. 